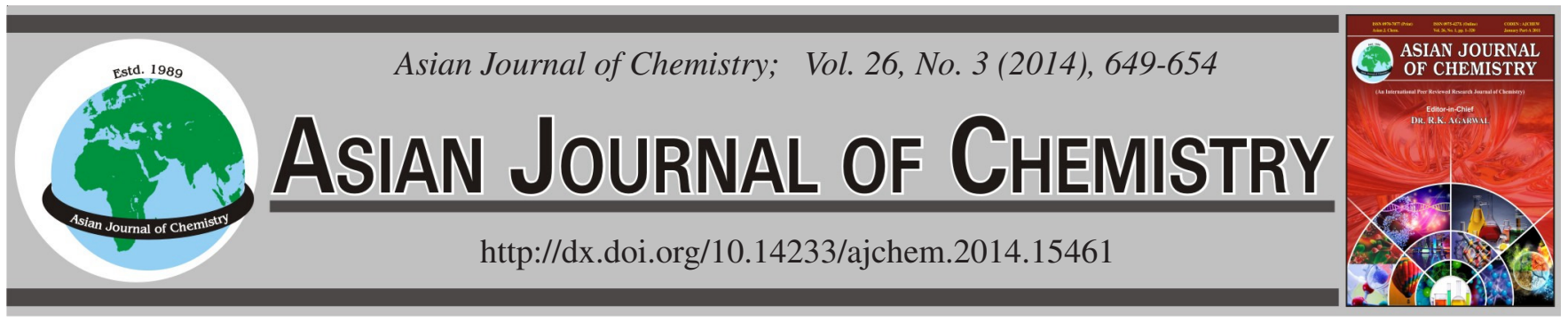

\title{
Structural Investigation on Pharmaceutical Intermediate $o$-Chlorobenzoyl Chloride: A DFT Approach
}

\author{
G. RAJA ${ }^{1, *}$, K. SARAVANAN ${ }^{2}$ and S. SivaKumar ${ }^{3}$
}

${ }^{1}$ Department of Chemistry, Paavai Engineering College, Namakkal-637 018, India

${ }^{2}$ Department of Chemistry, Thiruvalluvar Government Arts College, Rasipuram-637 401, India

${ }^{3}$ Department of Physics, Government Arts Collge (Autonomous), Salem-636 007, India

*Corresponding author: E-mail: genuineraja@gmail.com

Received: 9 March 2013;

Accepted: 5 August 2013;

Published online: 30 January 2014;

AJC-14607

The FT-IR and FT-Raman spectra of $o$-chlorobenzoyl chloride were recorded in the regions $4000-400 \mathrm{~cm}^{-1}$ and $3500-100 \mathrm{~cm}^{-1}$. The fundamental vibrational frequencies and intensity of vibrational bands were evaluated using density functional theory (DFT) and standard B3LYP/6-311+G** basis set combination. The vibrational spectra were interpreted, with the aid of normal coordinate analysis based on a scaled quantum mechanical (SQM) force field. The infrared and Raman spectra were also predicted from the calculated intensities. Comparison of simulated spectra with the experimental spectra provides important information about the ability of the computational method to describe the vibrational modes. Further, density functional theory (DFT) combined with quantum chemical calculations to determine the first-order hyperpolarizability.

Keywords: Vibrational spectra, FT-IR and FT-Raman spectra, DFT calculation, First-order hyperpolarizability.

\section{INTRODUCTION}

Quantum chemical computational methods have proved to be an essential tool for interpreting and predicting the vibrational spectra ${ }^{1,2}$. A significant advancement in this area was made by combining semi empirical quantum mechanical method; $a b$ initio quantum mechanical method and density functional theory (DFT), each method having its own advantage $^{3-6}$. In scaled quantum mechanical (SQM) approach, the systematic errors of the computed harmonic force field are corrected by a few scale factors which are found to be well transferable between chemically related molecules ${ }^{2,7,8}$ and were recommended for general use. In the present investigation, the vibrational analysis of $o$-chlorobenzoyl chloride using the SQM force field method based on DFT calculation was presented. The infrared and Raman spectra of the $o$-chlorobenzoyl chloride were also simulated utilizing the computed dipole derivatives for IR and polarizability derivatives for Raman activities. Further, we adopted density functional theory (DFT) combined with quantum chemical calculations to determine the first-order hyperpolarizability.

\section{EXPERIMENTAL}

Spectroscopically pure $o$-chlorobenzoyl chloride was obtained from Lancaster chemical company, U.K. and used as such for the spectral measurements. The room temperature Fourier transform infrared spectra of $o$-chlorobenzoyl chloride was recorded in the $4000-400 \mathrm{~cm}^{-1}$ region at a resolution of $\pm 1 \mathrm{~cm}^{-1}$, using BRUKER IFS-66V vacuum Fourier transform spectrometer, equipped with an MCT detector, a $\mathrm{KBr}$ beam splitter and globar source. The FT-Raman spectrum was recorded on the same instrument with FRA-106 Raman accessories in the region $3500-100 \mathrm{~cm}^{-1}$. Nd:YAG laser operating at $200 \mathrm{~mW}$ power with $1064 \mathrm{~nm}$ excitation was used as source.

Computational details: In order to find the most optimized molecular geometry, the energy and vibrational frequency calculations were carried out for $o$-chlorobenzoyl chloride with GAUSSIAN 98W software package ${ }^{9}$ using the B3LYP functional $^{10,11}$ standard $6-311+\mathrm{G}^{* *}$ (large) basis sets. The Cartesian representation of the theoretical force constants have been computed at optimized geometry by assuming $\mathrm{C}_{\mathrm{s}}$ point group symmetry. Scaling of the force field was performed according to the SQM procedure ${ }^{12,13}$ using selective scaling in the natural internal coordinate representation ${ }^{14,15}$. Transformation of the force field and subsequent normal coordinate analysis including the least square refinement of the scale factors, calculation of the total energy distribution (TED) and the prediction of IR and Raman intensities were done on a PC with the MOLVIB program (version 7.0-G77) written by Sundius ${ }^{16,17,18}$. For the plots of simulated IR and Raman spectra, 
pure Lorentizian band shapes were used with a band width of $10 \mathrm{~cm}^{-1}$.

The symmetry of the molecule was also helpful in making vibrational assignments. By combining the results of the GAUSSVIEW program ${ }^{19}$ with symmetry considerations, vibrational frequency assignments were made with a high degree of confidence. The defined local symmetry coordinates ${ }^{20-23}$ form complete set and matches quite well with the motions observed using the GAUSSVIEW program ${ }^{19}$.

\section{RESULTS AND DISCUSSION}

Molecular geometry: The optimized molecular structure of $o$-chlorobenzoyl chloride was shown in Fig. 1. The global minimum energy obtained by the DFT structure optimization was presented in Table-1. The optimized geometrical parameters obtained by the large basis set calculation were presented in Table-2.

Detailed description of vibrational modes can be given by means of normal coordinate analysis (NCA). For this purpose,

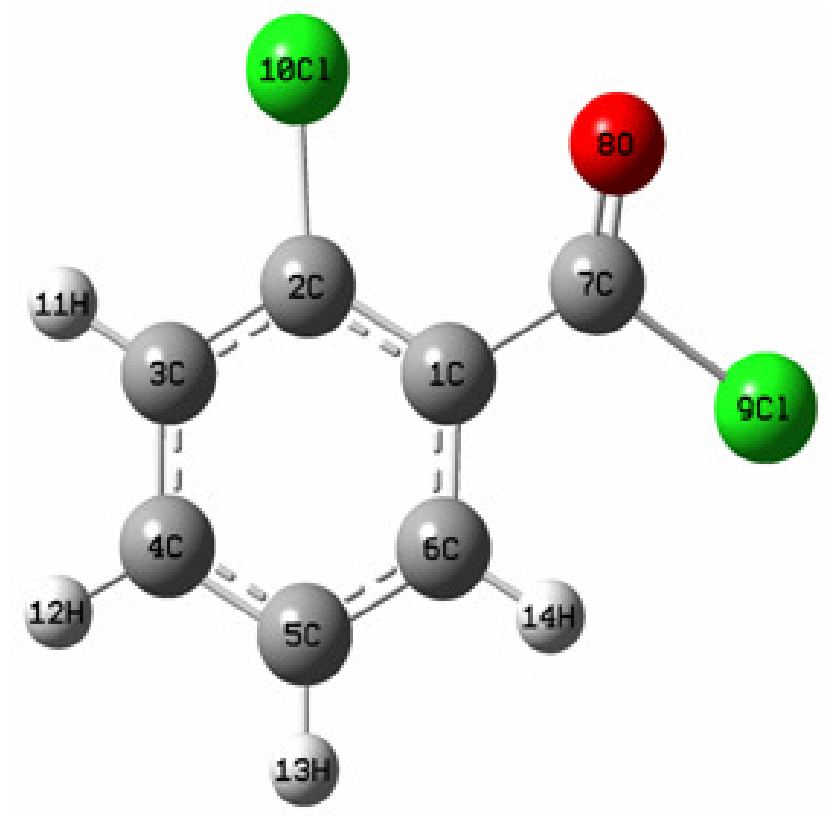

Fig. 1. The optimized molecular structure of $o$-chlorobenzoyl chloride

\begin{tabular}{|c|c|}
\hline \multicolumn{2}{|c|}{$\begin{array}{c}\text { TABLE-1 } \\
\text { TOTAL ENERGIES OF } o \text {-CHLOROBENZOYL CHLORIDE } \\
\text { CALCULATED AT DFT (B3LYP)/6-31G } \text { F }^{*} \text { AND } \\
\text { (B3LYP)/6-311 + G** LEVEL }\end{array}$} \\
\hline Method & Energies (Hartrees) \\
\hline $6-31 \mathrm{G}^{*}$ & -1264.54843900 \\
\hline $6-311+G^{* *}$ & -1264.76957264 \\
\hline
\end{tabular}

the full set of 48 standard internal coordinates containing 12 redundancies were defined as given in Table-3. From these, a non-redundant set of local symmetry coordinates were constructed by suitable linear combinations of internal coordinates following the recommendations of Fogarasi et. $a l^{5,6,14,15}$ are summarized in Table-4. The theoretically calculated DFT force fields were transformed in this later set of vibrational coordinates and used in all subsequent calculations.

Analysis of vibrational spectra: The 36 normal modes of $o$-chlorobenzoyl chloride are distributed among the symmetry species as $\Gamma_{3 \mathrm{~N}-6}=25 \mathrm{~A}^{\prime}$ (in-plane) $+11 \mathrm{~A}^{\prime \prime}$ (out-of-plane) and in agreement with $\mathrm{C}_{\mathrm{s}}$ symmetry. All the vibrations were active both in Raman scattering and infrared absorption. In the Raman spectrum the in-plane vibrations $\left(\mathrm{A}^{\prime}\right)$ give rise to polarized bands while the out-of-plane ones $\left(\mathrm{A}^{\prime \prime}\right)$ to depolarized band.

The detailed vibrational assignments of fundamental modes of $o$-chlorobenzoyl chloride along with calculated IR, Raman intensities and normal mode descriptions (characterized by TED) were reported in Table-5. For visual comparison, the observed and simulated FT-IR and FT-Raman spectra of $o$ chlorobenzoyl chloride are produced in a common frequency scales in Fig. 2 and 3.

Root mean square (RMS) values of frequencies were obtained in the study using the following expression,

$$
\mathrm{RMS}=\sqrt{\frac{1}{\mathrm{n}-1} \sum_{\mathrm{i}}^{\mathrm{n}}\left(\mathrm{v}_{\mathrm{i}}^{\text {calc }}-v_{\mathrm{i}}^{\text {exp }}\right)^{2}}
$$

The RMS error of the observed and calculated frequencies (unscaled / B3LYP/6-311 $+\mathrm{G}^{* *}$ ) of $o$-chlorobenzoyl chloride was found to be $109 \mathrm{~cm}^{-1}$. This is quite obvious; since the frequencies calculated on the basis of quantum mechanical force fields usually differ appreciably from observed frequencies. This is partly due to the neglect of anharmonicity and

\begin{tabular}{|c|c|c|c|c|c|}
\hline \multicolumn{6}{|c|}{$\begin{array}{c}\text { TABLE-2 } \\
\text { OPTIMIZED GEOMETRICAL PARAMETERS OF } o \text {-CHLOROBENZOYL CHLORIDE OBTAINED BY } \\
\text { B3LYP/ } 6-311+\mathrm{G}^{* *} \text { DENSITY FUNCTIONAL CALCULATIONS }\end{array}$} \\
\hline Bond length & Value $(\AA)$ & Bond angle & Value $(\AA)$ & Dihedral angle & Value $(\AA)$ \\
\hline $\mathrm{C} 2-\mathrm{C} 1$ & 1.41414 & $\mathrm{C} 3-\mathrm{C} 2-\mathrm{C} 1$ & 120.3423 & C4-C3-C2-C1 & 0.98560 \\
\hline $\mathrm{C} 3-\mathrm{C} 2$ & 1.39565 & $\mathrm{C} 4-\mathrm{C} 3-\mathrm{C} 2$ & 120.34093 & $\mathrm{C} 5-\mathrm{C} 4-\mathrm{C} 3-\mathrm{C} 2$ & -0.65574 \\
\hline $\mathrm{C} 4-\mathrm{C} 3$ & 1.39287 & $\mathrm{C} 5-\mathrm{C} 4-\mathrm{C} 3$ & 120.12402 & $\mathrm{C} 6-\mathrm{C} 5-\mathrm{C} 4-\mathrm{C} 3$ & -0.29085 \\
\hline $\mathrm{C} 5-\mathrm{C} 4$ & 1.39512 & C6-C5-C4 & 119.62347 & C7-C1-C6-C5 & 177.02637 \\
\hline C6-C5 & 1.38942 & $\mathrm{C} 7-\mathrm{C} 1-\mathrm{C} 6$ & 120.69086 & $\mathrm{O} 8-\mathrm{C} 7-\mathrm{C} 1-\mathrm{C} 6$ & -155.47786 \\
\hline $\mathrm{C} 7-\mathrm{C} 1$ & 1.48656 & $\mathrm{O} 8-\mathrm{C} 7-\mathrm{C} 1$ & 127.66669 & $\mathrm{C} 19-\mathrm{C} 7-\mathrm{C} 1-\mathrm{C} 6$ & 24.83177 \\
\hline $\mathrm{O} 8-\mathrm{C} 7$ & 1.18951 & $\mathrm{C} 19-\mathrm{C} 7-\mathrm{C} 1$ & 114.17689 & $\mathrm{Cl} 10-\mathrm{C} 2-\mathrm{C} 1-\mathrm{C} 6$ & -178.44100 \\
\hline $\mathrm{Cl} 9-\mathrm{C} 7$ & 1.84426 & $\mathrm{Cl} 10-\mathrm{C} 2-\mathrm{C} 1$ & 122.98120 & $\mathrm{H} 11-\mathrm{C} 3-\mathrm{C} 2-\mathrm{C} 1$ & -179.02943 \\
\hline $\mathrm{Cl} 10-\mathrm{C} 2$ & 1.74763 & $\mathrm{H} 11-\mathrm{C} 3-\mathrm{C} 2$ & 118.93682 & $\mathrm{H} 12-\mathrm{C} 4-\mathrm{C} 3-\mathrm{C} 2$ & 179.56864 \\
\hline H11-C3 & 1.08446 & $\mathrm{H} 12-\mathrm{C} 4-\mathrm{C} 3$ & 119.43315 & $\mathrm{H} 13-\mathrm{C} 5-\mathrm{C} 4-\mathrm{C} 3$ & 179.97229 \\
\hline $\mathrm{H} 12-\mathrm{C} 4$ & 1.08623 & H13-C5-C4 & 120.56340 & H14-C6-C5-C4 & -179.71471 \\
\hline $\mathrm{H} 13-\mathrm{C} 5$ & 1.08540 & H14-C6-C5 & 119.93207 & - & - \\
\hline H14-C6 & 1.08284 & - & - & - & - \\
\hline
\end{tabular}




\begin{tabular}{|c|c|c|c|}
\hline \multicolumn{4}{|c|}{$\begin{array}{c}\text { TABLE-3 } \\
\text { DEFINITION OF INTERNAL COORDINATES OF } o \text {-CHLOROBENZOYL CHLORIDE }\end{array}$} \\
\hline No (i) & Symbol & Type & Definition \\
\hline Streching 1-4 & $r_{i}$ & $\mathrm{C}-\mathrm{H}$ (arom.) & C3-H11, C4-H12, C5-H13, C6-H14 \\
\hline 5 & $\mathrm{R}_{\mathrm{i}}$ & $\mathrm{C}-\mathrm{Cl}$ (arom.) & $\mathrm{C} 2-\mathrm{C} 110$ \\
\hline 6 & $\mathrm{R}_{\mathrm{i}}$ & $\mathrm{C}-\mathrm{Cl}$ (sub.) & $\mathrm{C} 7-\mathrm{Cl} 9$ \\
\hline 7 & $\mathrm{p}_{\mathrm{i}}$ & $\mathrm{C}-\mathrm{O}$ (sub.) & $\mathrm{C} 7-\mathrm{O} 8$ \\
\hline $8-13$ & $P_{i}$ & C-C (ring) & $\mathrm{C} 1-\mathrm{C} 2, \mathrm{C} 2-\mathrm{C} 3, \mathrm{C} 3-\mathrm{C} 4, \mathrm{C} 4-\mathrm{C} 5, \mathrm{C} 5-\mathrm{C} 6, \mathrm{C} 6-\mathrm{C} 1$ \\
\hline 14 & $P_{i}$ & C-C (sub.) & $\mathrm{C} 7-\mathrm{C} 1$ \\
\hline Bending $15-22$ & $\theta_{\mathrm{i}}$ & $\mathrm{C}-\mathrm{C}-\mathrm{H}$ & $\begin{array}{l}\text { C2-C3-H11, C4-C3-H11, C3-C4-H12, C5-C4-H12, C4-C5-H13, } \\
\text { C6-C5-H13, C5-C6-H14, C1-C6-H14 }\end{array}$ \\
\hline 23 & $\beta_{\mathrm{i}}$ & $\mathrm{C}-\mathrm{C}-\mathrm{Cl}$ & $\mathrm{C} 1-\mathrm{C} 7-\mathrm{Cl} 9$ \\
\hline $24-25$ & $\beta_{\mathrm{i}}$ & $\mathrm{C}-\mathrm{C}-\mathrm{Cl}$ & $\mathrm{C} 1-\mathrm{C} 2-\mathrm{Cl} 10, \mathrm{C} 3-\mathrm{C} 2-\mathrm{Cl} 10$ \\
\hline 26 & $\gamma_{\mathrm{i}}$ & $\mathrm{C}-\mathrm{C}-\mathrm{O}$ & $\mathrm{C} 1-\mathrm{C} 7-\mathrm{O} 8$ \\
\hline $27-28$ & $\alpha_{i}$ & $\mathrm{C}-\mathrm{C}-\mathrm{C}$ & C6-C1-C7, C2-C1-C7 \\
\hline $29-34$ & $\alpha_{i}$ & C-C-C (ring) & $\begin{array}{l}\mathrm{C} 1-\mathrm{C} 2-\mathrm{C} 3, \mathrm{C} 2-\mathrm{C} 3-\mathrm{C} 4, \mathrm{C} 3-\mathrm{C} 4-\mathrm{C} 5 \\
\mathrm{C} 4-\mathrm{C} 5-\mathrm{C} 6, \mathrm{C} 5-\mathrm{C} 6-\mathrm{C} 1, \mathrm{C} 6-\mathrm{C} 1-\mathrm{C} 2\end{array}$ \\
\hline Out-of-plane $35-38$ & $\omega_{\mathrm{i}}$ & $\mathrm{C}-\mathrm{H}$ & $\begin{array}{l}\text { H11-C3-C2-C4, H12-C4-C3-C5, } \\
\text { H13-C5-C4-C6, H14-C6-C5-C1 }\end{array}$ \\
\hline 39 & $\omega_{\mathrm{i}}$ & $\mathrm{C}-\mathrm{Cl}$ & $\mathrm{Cl} 10-\mathrm{C} 2-\mathrm{C} 1-\mathrm{C} 3$ \\
\hline 40 & $\omega_{i}$ & $\mathrm{C}-\mathrm{C}$ & C7-C1-C2-C6 \\
\hline 41 & $\omega_{i}$ & $\mathrm{C}-\mathrm{Cl}$ & $\mathrm{C} 1-\mathrm{C} 7-\mathrm{O} 8-\mathrm{Cl} 9$ \\
\hline Torison $42-47$ & $\tau_{\mathrm{i}}$ & tring & $\begin{array}{l}\mathrm{C} 1-\mathrm{C} 2-\mathrm{C} 3-\mathrm{C} 4, \mathrm{C} 2-\mathrm{C} 3-\mathrm{C} 4-\mathrm{C} 5 \\
\mathrm{C} 3-\mathrm{C} 4-\mathrm{C} 5-\mathrm{C} 6, \mathrm{C} 4-\mathrm{C} 5-\mathrm{C} 6-\mathrm{C} 1, \\
\mathrm{C} 5-\mathrm{C} 6-\mathrm{C} 1-\mathrm{C} 2, \mathrm{C} 6-\mathrm{C} 1-\mathrm{C} 2-\mathrm{C} 3\end{array}$ \\
\hline 48 & $\tau_{\mathrm{i}}$ & $\tau \mathrm{C}-\mathrm{Cl}$ & $\mathrm{C} 2(\mathrm{C} 6)-\mathrm{C} 1-\mathrm{C} 7-\mathrm{O} 8(\mathrm{Cl})$ \\
\hline
\end{tabular}

TABLE-4

DEFINITION OF LOCAL SYMMETRY COORDINATES AND THE VALUE CORRESPONDING SCALE FACTORS USED TO CORRECT THE FORCE FIELDS FOR $o$-CHLOROBENZOYL CHLORIDE

\begin{tabular}{|c|c|c|c|}
\hline No.(i) & Symbol $^{\mathrm{a}}$ & Definition $^{\mathrm{b}}$ & Scale factors used in calculation \\
\hline $1-4$ & $\mathrm{C}-\mathrm{H}$ & $\mathrm{r} 1, \mathrm{r} 2, \mathrm{r} 3, \mathrm{r} 4$ & 0.920 \\
\hline 5 & $\mathrm{C}-\mathrm{Cl}$ (arom.) & R5 & 0.927 \\
\hline 6 & $\mathrm{C}-\mathrm{Cl}$ (sub.) & R6 & 0.927 \\
\hline 7 & $\mathrm{C}-\mathrm{O}$ & p7 & 0.918 \\
\hline $8-13$ & $\mathrm{C}-\mathrm{C}$ & P8, P9, P10, P11, P12, P13 & 0.902 \\
\hline 14 & C-C (sub.) & P14 & 0.902 \\
\hline $15-18$ & C-C-H & $\begin{array}{l}(\theta 15-\theta 16) / \sqrt{ } 2,(\theta 17-\theta 18) / \sqrt{ } 2 \\
(\theta 19-\theta 20) / \sqrt{ } 2,(\theta 21-\theta 22) / \sqrt{ } 2\end{array}$ & 0.942 \\
\hline 19 & $\mathrm{C}-\mathrm{C}-\mathrm{Cl}$ & $\beta 23$ & 0.947 \\
\hline 20 & $\mathrm{C}-\mathrm{C}-\mathrm{Cl}$ & $(\beta 24-\beta 25) / \sqrt{ } 2$ & 0.947 \\
\hline 21 & $\mathrm{C}-\mathrm{C}-\mathrm{O}$ & $\gamma 26$ & 0.979 \\
\hline 22 & C-C-C & $(\alpha 27-\alpha 28) / \sqrt{ } 2$ & 0.986 \\
\hline 23 & $\mathrm{~b}$ ring & $(\alpha 29-\alpha 30+\alpha 31-\alpha 32+\alpha 33-\alpha 34) / \sqrt{ } 6$ & 0.986 \\
\hline 24 & $\mathrm{~b}$ ring & $(2 \alpha 29-\alpha 30-\alpha 31+2 \alpha 32-\alpha 33-\alpha 34) / \sqrt{ } 12$ & 0.986 \\
\hline 26 & $\mathrm{C}-\mathrm{H}$ & $\omega 35, \omega 36, \omega 37, \omega 38$ & 0.963 \\
\hline $27-30$ & $\mathrm{C}-\mathrm{Cl}$ & $\omega 39$ & 0.965 \\
\hline 31 & $\mathrm{C}-\mathrm{C}$ & $\omega 40$ & 0.935 \\
\hline 32 & $\mathrm{C}-\mathrm{Cl}$ & $\omega 41$ & 0.963 \\
\hline 33 & $\mathrm{t}$ ring & $(\tau 42-\tau 43+\tau 44-\tau 45+\tau 46-\tau 47) / \sqrt{ } 6$ & 0.935 \\
\hline 34 & t ring & $(\tau 42-\tau 44+\tau 45-\tau 47) / 2$ & 0.935 \\
\hline 35 & t ring & $(-\tau 42+2 \tau 43-\tau 44-\tau 45+2 \tau 46-\tau 47) / \sqrt{ } 12$ & 0.935 \\
\hline 36 & $\tau \mathrm{C}-\mathrm{Cl}$ & $\tau 48 / 4$ & 0.945 \\
\hline
\end{tabular}

${ }^{a}$ These symbols are used for description of the normal modes by TED in Table- 5

$\mathrm{b}$ The internal coordinates used here are defined in Table- 3

partly due to the approximate nature of the quantum mechanical methods. In order to reduce the overall deviation between the unscaled and observed fundamental frequencies, scale factors were applied in the normal coordinate analysis and the subsequent least square fit refinement algorithm resulted into a very close agreement between the observed fundamentals and the scaled frequencies. Refinement of the scaling factors applied in this study achieved a weighted mean deviation of 9 $\mathrm{cm}^{-1}$ between the experimental and scaled frequencies of the $o$-chlorobenzoyl chloride. 
TABLE-5

DETAILED ASSIGNMENTS OF FUNDAMENTAL VIBRATIONS OF $\boldsymbol{o}$-CHLOROBENZOYL CHLORIDE BY NORMAL MODE ANALYSIS BASED ON SQM FORCE FIELD CALCULATION

\begin{tabular}{|c|c|c|c|c|c|c|c|c|}
\hline \multirow{2}{*}{ No. } & \multirow{2}{*}{$\begin{array}{c}\text { Symmetry } \\
\text { species } \\
\text { Cs }\end{array}$} & \multicolumn{2}{|c|}{$\begin{array}{l}\text { Observed frequency } \\
\left(\mathrm{cm}^{-1}\right)\end{array}$} & \multicolumn{4}{|c|}{$\begin{array}{l}\text { Calculated frequency }\left(\mathrm{cm}^{-1}\right) \text { with } \\
\text { B3LYP/6-311+G }{ }^{* *} \text { force field }\end{array}$} & \multirow[t]{2}{*}{ TED (\%) among type of internal coordinates ${ }^{c}$} \\
\hline & & Infrared & Raman & $\begin{array}{c}\text { Un } \\
\text { scaled }\end{array}$ & Scaled & $\operatorname{IR}^{\mathrm{a}} \mathrm{A}_{\mathrm{i}}$ & $\operatorname{Raman}^{\mathrm{b}} \mathrm{I}_{\mathrm{i}}$ & \\
\hline 1 & $\mathrm{~A}^{\prime}$ & $3113 \mathrm{w}$ & & 3162 & 3117 & 0.133 & 67.02 & $\mathrm{CH}(99)$ \\
\hline 2 & $\mathrm{~A}^{\prime}$ & $3097 \mathrm{w}$ & $3082 \mathrm{~s}$ & 3254 & 3095 & 0.103 & 91.11 & $\mathrm{CH}(99)$ \\
\hline 3 & $\mathrm{~A}^{\prime}$ & 3074 vw & & 3213 & 3070 & 0.345 & 22.70 & CH(99) \\
\hline 4 & $\mathrm{~A}^{\prime}$ & $3026 \mathrm{vw}$ & & 3194 & 3028 & 0.307 & 81.88 & CH(99) \\
\hline 5 & $\mathrm{~A}^{\prime}$ & 1942 w & & 2145 & 1947 & 0.732 & 76.02 & $\mathrm{CO}(47), \mathrm{gClO}(18), \mathrm{CC}(12), \operatorname{tCCl}(7), \mathrm{bCCl}(5)$ \\
\hline 6 & $\mathrm{~A}^{\prime}$ & 1786 vs & $1790 \mathrm{~s}$ & 1992 & 1791 & 0.127 & 63.50 & $\mathrm{CO}(68), \mathrm{bCH}(18)$, bring $(12)$ \\
\hline 7 & $\mathrm{~A}^{\prime}$ & $1638 \mathrm{~s}$ & & 1936 & 1641 & 0.937 & 23.18 & $\mathrm{CC}(68), \mathrm{bCH}(16), \operatorname{bring}(11)$ \\
\hline 8 & $A^{\prime \prime}$ & $1588 \mathrm{w}$ & 1592 vs & 1803 & 1601 & 0.796 & 1.599 & $\mathrm{CC}(50), \mathrm{bCH}(44)$ \\
\hline 9 & $\mathrm{~A}^{\prime}$ & $1568 \mathrm{~s}$ & $1574 \mathrm{~s}$ & 1773 & 1572 & 0.39 & 16.26 & $\mathrm{CC}(52), \mathrm{bCH}(35), \mathrm{bCC}(8)$ \\
\hline 10 & $A^{\prime \prime}$ & $1463 \mathrm{~s}$ & & 1623 & 1467 & 0.654 & 3.34 & $\mathrm{CC}(89)$ \\
\hline 11 & $\mathrm{~A}^{\prime}$ & $1439 \mathrm{~s}$ & & 1672 & 1442 & 0.486 & 16.42 & bCH(66), CC(19), bCC(8) \\
\hline 13 & $\mathrm{~A}^{\prime}$ & 1287 vs & & 1514 & 1292 & 0.324 & 17.87 & bCH(86), CC(13) \\
\hline 14 & $A^{\prime \prime}$ & $1267 \mathrm{~s}$ & & 1472 & 1271 & 0.207 & 6.41 & $\mathrm{CC}(41), \mathrm{bCH}(38), \mathrm{CCl}(9), \mathrm{bCC}(8)$ \\
\hline 15 & $A^{\prime \prime}$ & $1192 \mathrm{~s}$ & $1196 \mathrm{~s}$ & 1388 & 1196 & 0.028 & 5.19 & bring(47), $\mathrm{CC}(23), \mathrm{CCl}(17), \mathrm{bCH}(10)$ \\
\hline 16 & $\mathrm{~A}^{\prime}$ & $1166 \mathrm{~s}$ & $1171 \mathrm{~s}$ & 1377 & 1169 & 0.082 & 7.43 & bring(77), bCH(19) \\
\hline 17 & $\mathrm{~A}^{\prime}$ & $1138 \mathrm{~s}$ & $1142 \mathrm{~s}$ & 1342 & 1142 & 0.723 & 4.28 & $\operatorname{tring}(83), \mathrm{gCH}(16)$ \\
\hline 18 & $\mathrm{~A}^{\prime}$ & $1067 \mathrm{~s}$ & & 1317 & 1069 & 0.558 & 8.39 & $\operatorname{tring}(81), \mathrm{gCH}(11), \mathrm{gCC}(5)$ \\
\hline 19 & $\mathrm{~A}^{\prime}$ & $1043 \mathrm{~s}$ & $1047 \mathrm{~s}$ & 1243 & 1046 & 0.744 & 1.14 & $\operatorname{tring}(70), \mathrm{CO}(6), \mathrm{gCC}(6), \mathrm{gCH}(6)$ \\
\hline 20 & $A^{\prime \prime}$ & 957 vs & & 1047 & 959 & 0.155 & 6.99 & $\mathrm{~b}$ ring(21), $\mathrm{CC}(21), \mathrm{bCO}(14), \mathrm{gClO}(14), \mathrm{gCH}(7), \mathrm{CCl}(6)$ \\
\hline 21 & $A^{\prime \prime}$ & $866 \mathrm{~s}$ & & 1038 & 870 & 0.996 & 6.11 & $\mathrm{gCH}(50), \mathrm{gCC}(24), \operatorname{tring}(11), \mathrm{bCO}(6), \mathrm{bCCl}(5)$ \\
\hline 22 & $A^{\prime \prime}$ & $766 \mathrm{~s}$ & & 921 & 768 & 0.06 & 0.86 & $\mathrm{gCCl}(26), \mathrm{gCC}(24)$, bring $(12), \mathrm{bCO}(9), \mathrm{gCH}(7), \operatorname{tring}(7)$ \\
\hline 23 & $\mathrm{~A}^{\prime}$ & $726 \mathrm{~s}$ & & 942 & 729 & 0.03 & 3.54 & $\mathrm{gCCl}(31), \mathrm{gCC}(19)$, bring(10), gCH(9), tring(9), $\mathrm{ClO}(6)$ \\
\hline 24 & $\mathrm{~A}^{\prime}$ & $707 \mathrm{~s}$ & & 901 & 711 & 0.351 & 11.43 & $\operatorname{gCCl}(36), \mathrm{gClO}(27), \operatorname{bring}(9), \mathrm{bCC}(6)$ \\
\hline 25 & $\mathrm{~A}^{\prime}$ & $668 s$ & $664 s$ & 872 & 671 & 0.147 & 0.62 & $\mathrm{bCCl}(23), \mathrm{CO}(18), \mathrm{gClO}(18), \mathrm{bCO}(13), \operatorname{tring}(6), \mathrm{gCH}(5)$ \\
\hline 26 & $A^{\prime \prime}$ & $642 \mathrm{~s}$ & $647 w$ & 832 & 647 & 0.86 & 1.16 & bCCl(20), tring(11), $\mathrm{CC}(11), \mathrm{gCC}(10), \mathrm{gClO}(8), \mathrm{bCC}(8)$ \\
\hline 27 & $\mathrm{~A}^{\prime}$ & & $545 \mathrm{~s}$ & 743 & 548 & 0.79 & 5.65 & tring(38), $\mathrm{gCCl}(25), \mathrm{gCH}(9), \mathrm{CO}(7), \mathrm{tCCl}(6)$ \\
\hline 30 & $A^{\prime \prime}$ & $440 \mathrm{~s}$ & & 598 & 445 & 0.143 & 0.12 & $\mathrm{bCCl}(28), \operatorname{tring}(16), \mathrm{CO}(13), \mathrm{bCC}(10), \mathrm{gClO}(7)$ \\
\hline 31 & $\mathrm{~A}^{\prime}$ & & $431 \mathrm{~s}$ & 586 & 436 & 0.876 & 4.78 & $\operatorname{tCCl}(18), \mathrm{CO}(16), \mathrm{bCCl}(12), \mathrm{bCO}(12)$, bring $(12), \mathrm{CC}(10)$ \\
\hline 32 & $\mathrm{~A}^{\prime}$ & & $363 \mathrm{w}$ & 547 & 367 & 0.10 & 1.36 & $\mathrm{bCCl}(42), \mathrm{CO}(12), \mathrm{gCC}(10), \mathrm{gCCl}(9), \operatorname{tring}(7)$ \\
\hline 33 & $A^{\prime}$ & & $260 \mathrm{~s}$ & 428 & 266 & 0.103 & 2.43 & $\mathrm{CO}(34), \mathrm{gCC}(24), \mathrm{gCCl}(14), \operatorname{tring}(13)$ \\
\hline 34 & $\mathrm{~A}^{\prime}$ & & $247 \mathrm{~s}$ & 412 & 253 & 0.141 & 0.53 & $\mathrm{CO}(47), \mathrm{bCCl}(18), \mathrm{bCC}(14), \mathrm{gClO}(10)$ \\
\hline 35 & $\mathrm{~A}^{\prime}$ & & $173 \mathrm{w}$ & 363 & 177 & 0.129 & 3.03 & $\operatorname{tring}(33), \mathrm{CCl}(21), \mathrm{CO}(15), \mathrm{gCC}(12), \mathrm{tCCl}(11)$ \\
\hline 36 & $\mathrm{~A}^{\prime}$ & & $141 \mathrm{w}$ & 244 & 145 & 0.35 & 0.28 & $\mathrm{tCCl}(40), \mathrm{CO}(37), \mathrm{CCl}(16)$ \\
\hline $\begin{array}{l}\text { Abbi } \\
{ }^{a} \text { Rel } \\
{ }^{b} \operatorname{Rel}\end{array}$ & $\begin{array}{l}\text { tions use } \\
\text { absorpti } \\
\text { Raman i } \\
\text { otations }\end{array}$ & $\begin{array}{l}\text {, bendir } \\
\text { ntensiti } \\
\text { isities c }\end{array}$ & $\begin{array}{l}\text {, waggin } \\
\text { ormalize } \\
\text { lated by }\end{array}$ & $\begin{array}{l}\text { tors } \\
\text { ith } \mathrm{h}\end{array}$ & s, str & $\begin{array}{l}s, \text { ve } \\
\text { orpti } \\
\text { o } 10\end{array}$ & ong; w, & k; vw, very weak \\
\hline
\end{tabular}

C-C vibrations: The bands between $1650-1480 \mathrm{~cm}^{-1}$ are assigned to $\mathrm{C}-\mathrm{C}$ stretching modes ${ }^{23}$. In the present study, the carbon stretching vibrations of the $o$-chlorobenzoyl chloride have been observed at $1638,1588,1568 \mathrm{~cm}^{-1}$ in the FT-IR and $1592,1574 \mathrm{~cm}^{-1}$ in FT-Raman spectrum and are presented in Table-5. These assignments are in good agreement with literature $^{24,25}$.

C-H vibrations: Aromatic compounds commonly exhibit multiple weak bands in the region $3100-3000 \mathrm{~cm}^{-1}$ due to aromatic $\mathrm{C}-\mathrm{H}$ stretching vibrations. The bands due to $\mathrm{C}-\mathrm{H}$ in-plane ring bending vibration interacting with $\mathrm{C}-\mathrm{C}$ stretching vibration are observed as a number of $\mathrm{m}-\mathrm{w}$ intensity sharp bands in the region 1300-1000 $\mathrm{cm}^{-1}$. C-H out-of-plane bending vibrations are strongly coupled vibrations and occur in the region $900-667 \mathrm{~cm}^{-126}$. Accordingly, in the present study the
C-H vibrations of the title compounds are observed at 3097, $3074,3026 \mathrm{~cm}^{-1}$ in the FT-IR spectrum and 3082 in FT-Raman for $o$-chlorobenzoyl chloride.

C-Cl vibrations: $\mathrm{C}-\mathrm{Cl}$ vibrations are found in the region below $1000 \mathrm{~cm}^{-1}$. The $\mathrm{C}-\mathrm{Cl}$ stretching frequency is generally observed in the region $800-600 \mathrm{~cm}^{-1}$ depending on the configuration and conformation of the compound ${ }^{27-28}$. Based on this, the FT-IR bands at 766, 726, 707, 668, $642 \mathrm{~cm}^{-1}$ and FTRaman bands at $664,647 \mathrm{~cm}^{-1}$ have been assigned to $\mathrm{C}-\mathrm{Cl}$ stretching in $o$-chlorobenzoyl chloride.

C-O vibrations: The carbonyl stretching generally occurs as a strong absorption in the region from 1730 to $1645 \mathrm{~cm}^{-1}$. This portion of the infrared spectrum is most useful because the position of the carbonyl absorption is quite sensitive to substitution effects and the geometry of the molecule. Accordingly, 


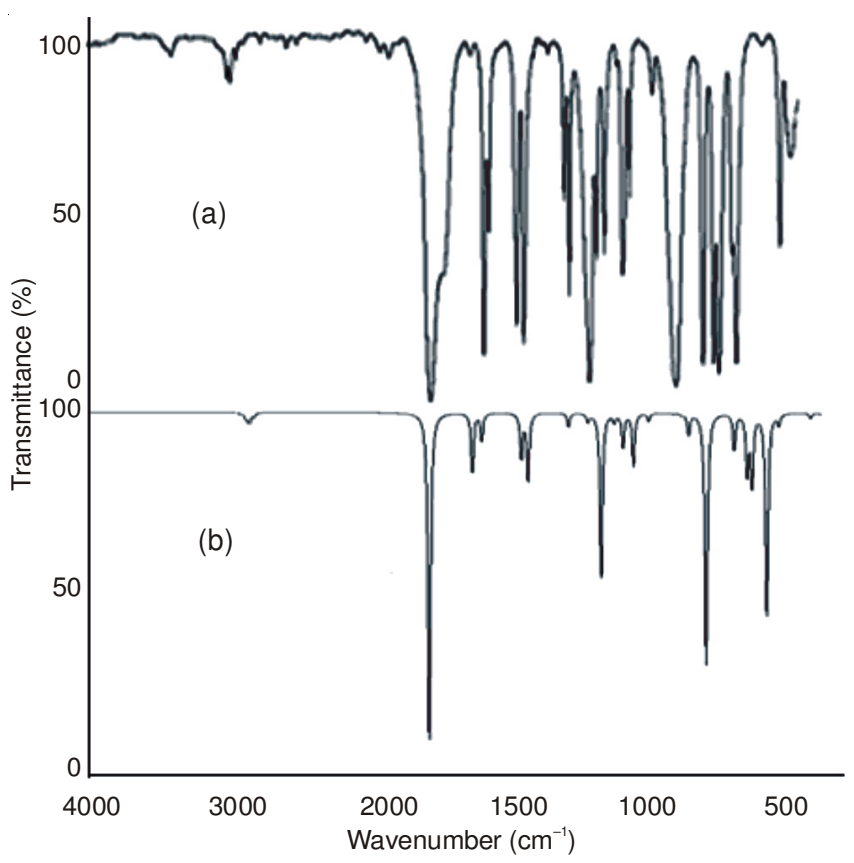

Fig. 2. FT-IR spectra of $o$-chlorobenzoyl chloride (a) observed (b) calculated with B3LYP/6-311+G**

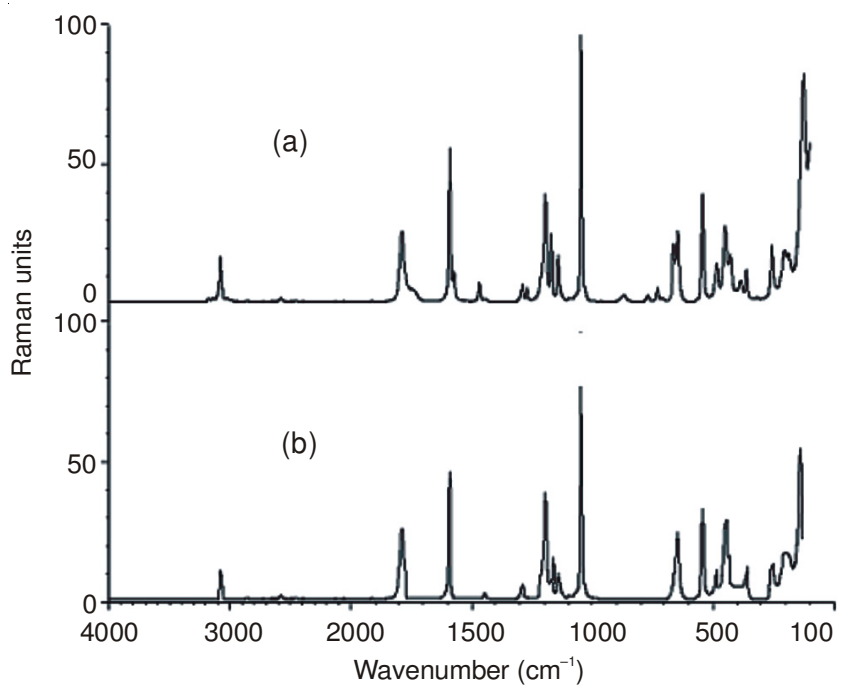

Fig. 3. FT-Raman spectra of $o$-chlorobenzoyl chloride (a) observed (b) calculated with B3LYP/6-311+G**

in the present investigation, the peaks identified at 1790 and $1786 \mathrm{~cm}^{-1}$ have been assigned to $\mathrm{C}=\mathrm{O}$ stretching vibrations for $o$-chlorobenzoyl chloride.

Ring vibrations: Many ring modes are affected by the substitution in the aromatic ring. In the present study, the bands absorbed at 1192, 1166, 1138, 1067, 1043, 957, $866 \mathrm{~cm}^{-1}$ and $486,440 \mathrm{~cm}^{-1}$ have been designated to ring in-plane and outof-plane bending modes, respectively.

Hyperpolarizability calculations: The first-order hyperpolarizibility $\left(\beta_{\mathrm{ijk}}\right)$ of the novel molecular system of $o$-chlorobenzoyl chloride is calculated using 3-21 G (d,p) basis set based on finite field approach. Hyperpolarizibility is a third rank tensor that can be described by a $3 \times 3 \times 3$ matrix. It strongly depends on the method and basis set used. The 27 components of $3 \mathrm{D}$ matrix can be reduced to 10 components due to Kleinman symmetry ${ }^{29}$. The calculated first-order hyperpolarizability (btotal) of $o$-chlorobenzoyl chloride is $1.289537 \times 10^{-30} \mathrm{esu}$, which is nearly seven times that of urea $\left(0.1947 \times 10^{-30} \mathrm{esu}\right)$. The calculated dipole moment $(\mu)$ and first-order hyperpolarizibility $(\beta)$ are shown in Table-6.

\begin{tabular}{|c|c|}
\hline \multicolumn{2}{|c|}{$\begin{array}{c}\text { TABLE-6 } \\
\text { THE DIPOLE MOMENT }(\mu) \text { AND FIRST-ORDER } \\
\text { HYPERPOLARIZIBILITY }(\beta) \text { OF } o \text {-CHLOROBENZOYL } \\
\text { CHLORIDE DERIVED FROM DFT CALCULATIONS }\end{array}$} \\
\hline$\beta_{\mathrm{xxx}}$ & 24.6798306 \\
\hline$\beta_{\mathrm{xxy}}$ & 4.1106034 \\
\hline$\beta_{\mathrm{xyy}}$ & 2.7371947 \\
\hline$\beta_{\text {yyy }}$ & -3.5868028 \\
\hline$\beta_{\mathrm{zxx}}$ & -118.5181275 \\
\hline$\beta_{\mathrm{xyz}}$ & -0.7739399 \\
\hline$\beta_{\mathrm{zyy}}$ & 1.3003588 \\
\hline$\beta_{x z z}$ & -12.4290667 \\
\hline$\beta_{\mathrm{yzz}}$ & 5.030306 \\
\hline$\beta_{z z z}$ & -36.030306 \\
\hline$\beta_{\text {total }}$ & 1.289537 \\
\hline$\mu_{\mathrm{x}}$ & 2.1196756 \\
\hline$\mu_{\mathrm{y}}$ & 0.0228399 \\
\hline$\mu_{\mathrm{z}}$ & 0.570441 \\
\hline$\mu$ & 2.195211 \\
\hline
\end{tabular}

\section{Conclusion}

In this work, the SQM force field method based on DFT calculations at the B3LYP/6-311 $+\mathrm{G}^{* *}$ level have been carried out to analyze the vibrational frequencies of $o$-chlorobenzoyl chloride. Refinement of the scaling factors applied in this study achieved a weighted RMS deviation of $9 \mathrm{~cm}^{-1}$ between the experimental and scaled frequencies. This close agreement established between the experimental and scaled frequ-encies obtained using large basis set $\left(6-311+\mathrm{G}^{* * *}\right)$ calculations has proved to be more reliable and accurate than the calculations using lower basis sets. The first-order hyperpolarizibility $\left(\beta_{\mathrm{ijk}}\right)$ of the novel molecular system of $o$-chlorobenzoyl chloride is calculated using 3-21 G (d,p) basis set based on finite field approach. The calculated first-order hyperpolarizability $\left(\beta_{\text {total }}\right)$ of $o$-chlorobenzoyl chloride is $1.289537 \times 10^{-30} \mathrm{esu}$, which is nearly seven times that of urea $\left(0.1947 \times 10^{-30} \mathrm{esu}\right)$.

\section{REFERENCES}

1. W.J. Hehre, L. Random, Schleyer, P.V.R. and Pople, J.A. Ab Initio Molecular Orbital Theory, Wiley, New York. P.548 (1986).

2. C.E. Blom and C. Altona, Mol. Phys., 31, 1377 (1976).

3. C.C.J. Roothaan and M. Synek, Phys. Rev., 133, 1263 (1964).

4. G.R. De Mare, Y.N. Panchenko and C.W. Bock, J. Phys. Chem., 98, 1416 (1994).

5. G. Fogarasi and P. Pulay, in ed: J.R. Durig, Vibrational Spectra and Structure, vol. 14, Elsevier, Amsterdam, p. 125 (1985).

6. G. Fogarasi, X. Xhov, P.W. Taylor and P. Pulay, J. Am. Chem. Soc., 114, 8191(1992).

7. T. Ziegler, Chem. Rev., 91, 651 (1991).

8. G. Socrates, Infrared and Raman Characteristic Group FrequenciesTables and Charts, edn 3, John Wiley \& Sons, Chichester (2001).

9. M.J. Frisch, G.W. Trucks, H.B. Schlega, G.E. Scuseria, M.A. Robb, J.R. Cheesman, V.G. Zakrzewski, J.A. Montgomery Jr., R.E. Stratmann, J.C. Burant, S. Dapprich, J.M. Millam, A.D. Daniels, K.N. Kudin, M.C. Strain, O. Farkas, J. Tomasi, V. Barone, M. Cossi, R. Cammi, B. Mennucci, 
C. Pomelli, C. Adamo, S. Clifford, J. Ochterski, G.A. Petersson, P.Y. Ayala, Q. Cui, K. Morokuma, N. Roga, N. Salvador, J.J. Dannenberg, D.K. Malick, A.D. Rabuck, K. Rahavachari, J.B. Foresman, J. Cioslowski, J.V. Ortiz, A.G. Baboul, B.B. Stefanov, G. Liu, A. Liashenko, P. Piskorz, I. Komaromi, R. Gomperts, R.L. Martin, D.J. Fox, T. Keith, M.A. Al-Laham, C.Y. Penng, A. Nanayakkara, M. Challa-Combe, P.M.W. Gill, B. Johnson, W. Chen, M.W. Wong, J.L. Andres, C. Gonzalez, M. Head-Gordon, E.S. Replogle and J.A. Pople, Gaussian 98, Revision A 11.4, Gaussian Inc., Pittsburgh, PA (2002).

10. C. Lee, W. Yang and R.G. Parr, Phys. Rev. B., 37, 785 (1998).

11. A.D. Becke, J. Chem. Phys., 98, 5648 (1993).

12. W.O. George, J.E. Goodfield and W.F. Maddams, Spectrochim. Acta A, 41, 1243 (1985).

13. B.A. Hess Jr., J. Schaad, P. Carsky and R. Zahraduik, Chem. Rev., 86, 709 (1986).

14. P. Pulay, G. Fogarasi, G. Pongor, J.E. Boggs and A. Vargha, J. Am. Chem. Soc., 105, 7037 (1983).

15. P. Pulay, X. Zhou and G. Fogarasi, in ed: R. Fransto, NATO AS Series, Kluwer, Dordrecht, Vol. C, 406, p.99 (1993).

16. T. Sundius, J. Mol. Struct., 218, 321 (1990).

17. T. Sundius, Vib. Spectrosc., 29, 89 (2002).
18. T. Sundius, MOLVIB (v.7.0), Calculation of Harmonic Force Fields and Vibrational Modes of Molecules, QCPE Program No. 807 (2002).

19. A. Frisch, A.B. Nielson and A.J. Holder, Gaussview Users Mannual, Gaussian Inc., Pittsburgh, PA (2000).

20. V. Krishna kumar and R. John Xavier, Indian J. Pure Appl. Phys., 41, 95 (2003).

21. B. Lakshmaiah and G. Ramana Rao, J. Raman Spectrosc., 20, 439 (1989).

22. J. Mohan, Organic Spectroscopy-Principles and Applications, Narosa Publishing House, New Delhi, edn 2 (2001).

23. P.L. Polavarapu, J. Phys. Chem., 94, 8106 (1990).

24. P.N. Prasad and D.J. Williams, Introduction to Nonlinear Optical Effects in Molecules and Polymers, Wiley, New York (1991).

25. G. Rauhut and P. Pulay, J. Phys. Chem., 99, 3093 (1995).

26. D.N. Sathyanarayana, Vibrational Spectroscopy-Theory and Applications, New Age International (P) Limited Publishers, New Delhi, edn 2 (2004).

27. D.N. Shin, J.W. Hahn, K.H. Jung and T.K. Ha, J. Raman Spectrosc., 29, 245 (1998).

28. Y. Yamakita and M. Tasuni, J. Phys. Chem., 99, 8524 (1995).

29. D.A. Kleinman, Phys. Rev., 126, 1977 (1962). 